\title{
Recombination dynamics of single quantum dots in a fiber system at telecommunication wavelengths
}

\author{
Xiulai Xu ${ }^{\mathrm{a}, *}$, Frederic Brossard ${ }^{\mathrm{a}}$, Kiyotaka Hammura ${ }^{\mathrm{a}}$, David A. Williams ${ }^{\mathrm{a}}$, B. Alloing ${ }^{\mathrm{b}}$, \\ L.H. Li ${ }^{b}$, Andrea Fiore ${ }^{c}$ \\ ${ }^{a}$ Hitachi Cambridge Laboratory, Hitachi Europe Ltd., JJ Thomson Avenue, Cambridge CB3 OHE, UK \\ ${ }^{\mathrm{b}}$ Ecole Polytechnique Fédérale de Lausanne, Institute of Photonics and Quantum Electronics, Station 3, CH-1015 Lausanne, Switzerland

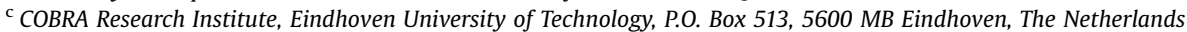

\section{A R T I C L E I N F O}

Available online 23 December 2008

PACS:

42.50.Dv

42.81.-i

78.67. Hc

Keywords:

‘Plug and play' single-photon sources

Lifetime dynamics

Telecommunication wavelengths

\begin{abstract}
A B S T R A C T
Coupling the emitted light from single quantum dots into an optic fiber has been demonstrated as a 'plug and play' single-photon source. In this work, lifetime dynamics measurements were performed with two time-delayed femtosecond optical pulses at different wavelengths. When the energy of the pumping light is less than the energy of the wetting layer (i.e. quasi-resonant excitation), the singlephoton sources can be operated at a speed of $1 \mathrm{GHz}$.
\end{abstract}

(c) 2008 Elsevier Ltd. All rights reserved.
Semiconductor quantum dot based single photon emission has been investigated intensively to implement quantum information processing [1-8]. Most investigations are concentrating on electrical pumping, high repetition rates, telecommunication wavelengths, high temperature operation and stability. We realized a 'plug and play' single photon sources fully integrated with optical fibers [2]. Great stability of nearly (but not limited to) one month has been demonstrated. Recently, the emission wavelength has been extended to telecommunication wavelengths using same system [9]. The details about the fiber-based system are described in Refs. [2,9]. To improve the repetition rates of the single photons for fast quantum key distribution system, photonic crystal and nano pillar cavities with distributed Bragg reflector (DBR) structure have been used to enhance emission rate, i.e. reducing lifetime of exciton recombination $[4,10]$. To count the single photons at telecommunication wavelengths, InGaAs single photon detectors are normally used. Because of the large dark counts of InGaAs detectors, the detectors are operated in Geiger mode and gated at $5 \mathrm{MHz}$ with a pulse width of $40 \mathrm{~ns}$. With this detector, it is difficult to verify how fast the single photon sources can be operated. To examine the pulse rate of our single-photon gun at $1.3 \mu \mathrm{m}$, time resolved photoluminescence (PL) measurements were performed with two separated optical pulses at different wavelengths.

\footnotetext{
* Corresponding author.

E-mail address: xx757@cam.ac.uk (X. Xu).
}

The InAs QD wafer with a $1.3 \mu \mathrm{m}$ wavelength emission is grown by molecular-beam epitaxy with an InGaAs capping layer. The QD layer is embedded in a 1-lambda cavity between 1-period (top) and 15-period (bottom) $\mathrm{GaAs} / \mathrm{Al}_{0.9} \mathrm{Ga}_{0.1} \mathrm{As} \mathrm{DBRs}$, in order to enhance the extraction efficiency. To achieve very low quantum dot density (around $2 \times 10^{8}$ dots $/ \mathrm{cm}^{2}$ ), an ultralow growth rate was used for the sample growth [11]. However, the dot density (around $10^{8}$ dots $/ \mathrm{cm}^{2}$ ) is still too high to isolate the light emission from a single quantum dot collected by a single mode fiber in the fiber bundle. To isolate single quantum dots for a single mode fiber, micro-pillars were patterned with electron beam lithography and lift-off techniques. An etch depth about $1-2 \mu \mathrm{m}$ is required to isolate the quantum dots because of the thickness of the top DBR mirrors. A layer of $\mathrm{SiO}_{2}$ has been used as the etching mask, which guarantees the etch depth. Fig. 1(a) shows the fabrication process of micro-pillars. At first, a layer of PMMA was spin-coated on the top of the quantum dot wafer. Holes with different sizes were patterned with electron beam writing. After the development, a layer of $\mathrm{SiO}_{2}$ was sputtered and lifted off, then reactive ion etching was performed. After etching, micro-pillars are formed with removal of the $\mathrm{SiO}_{2}$ layer, as shown in Fig. 1(b). With this patterned wafer, we have successfully achieved single dot emission using the fiber system [9].

Fig. 2 shows the lifetime measurement system. An optical pulse train with a pulse width of $100 \mathrm{fs}$ at $80 \mathrm{MHz}$ is generated from Ti:sapphire laser. The beam sampler takes about $10 \%$ of light intensity to the Si photodiode, which is connected to the time correlated single photon counting (TCSPC) card as a reference 
a

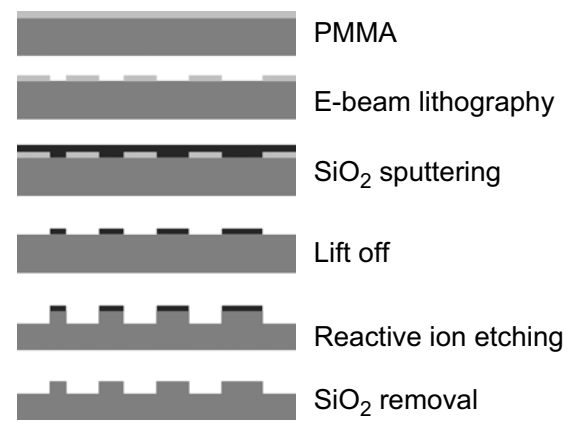

b

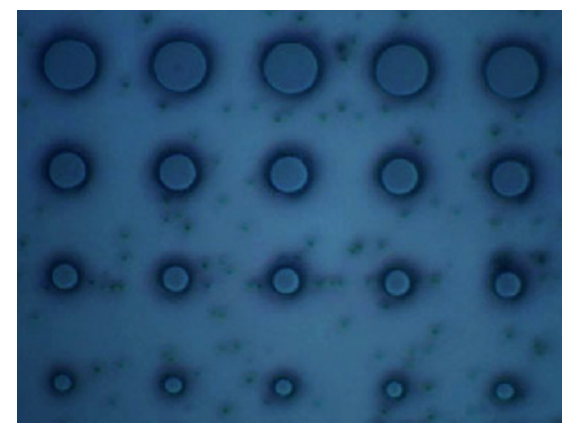

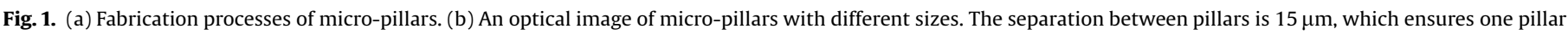
per single mode fiber.

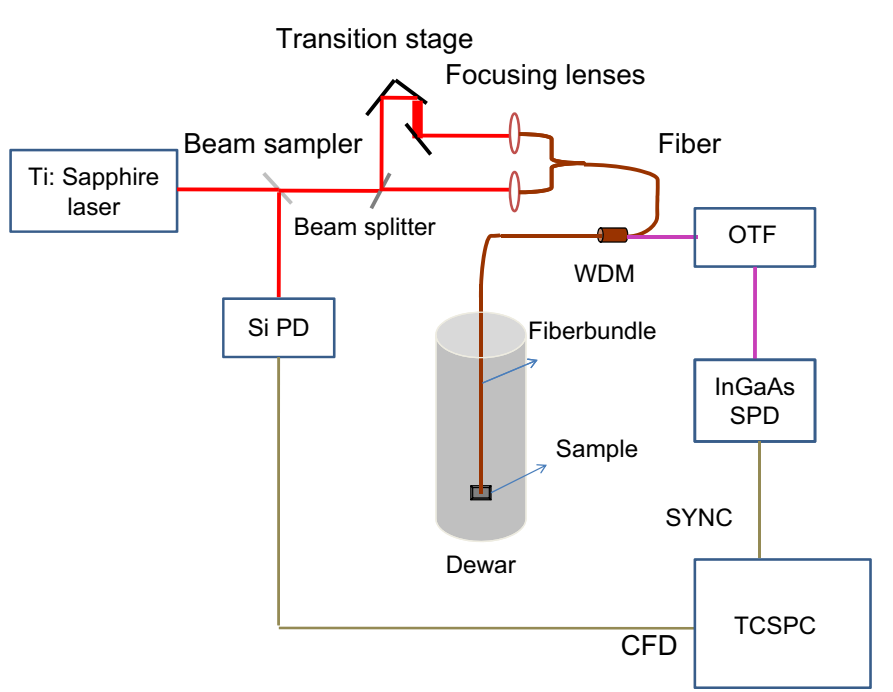

Fig. 2. Lifetime measurement setup for single quantum dots in a fiber system.

start. The rest beam is divided by a 50/50 beam splitter to two paths, one of the paths is delayed by a transition stage which controls the time delay between the two pulses. The two pulses are focused into the pumping fibers. The optical pulses excite the QD sample, mounted at the end of the fiber bundle and dipped in a liquid Helium dewar, via wavelength division multiplexing (WDM) coupler. The emitted light from quantum dots comes out through the output fiber via the WDM. To isolate an exciton line from other multi exciton emission, an optical tunable filter (OTF) with a band width of $0.75 \mathrm{~nm}$ is used. The inset in Fig. 3 shows a typical exciton line of a single quantum dot with an OTF. The single exciton emission is detected with the InGaAs single photon counter, the output from InGaAs detector is connected to the stop of the TCSPC. The resulting histograms show the decay dynamics of the exciton recombination.

The lifetime dynamics of the single dot emission is shown in Fig. 3. When the pumping energy (corresponding to a wavelength of $830 \mathrm{~nm}$ ) is larger than the wetting layer transition energy (around $870 \mathrm{~nm}$ ) [12], the lifetime is around $1.2 \pm 0.2 \mathrm{~ns}$. This is similar to other results on the similar quantum dot wafer [5]. If pumping quasi-resonantly, the lifetime of the quantum dots is about $0.7 \pm 0.2 \mathrm{~ns}$ (the top trace in Fig. 3). At high energy (nonresonant) pumping, the carriers generated in the GaAs surrounding the QDs may be trapped in local potential minima or surface states, then relax to the wetting layer first, to the excited states of quantum dots, subsequently to the ground states for recombination, resulting in a longer lifetime. The shorter lifetime with

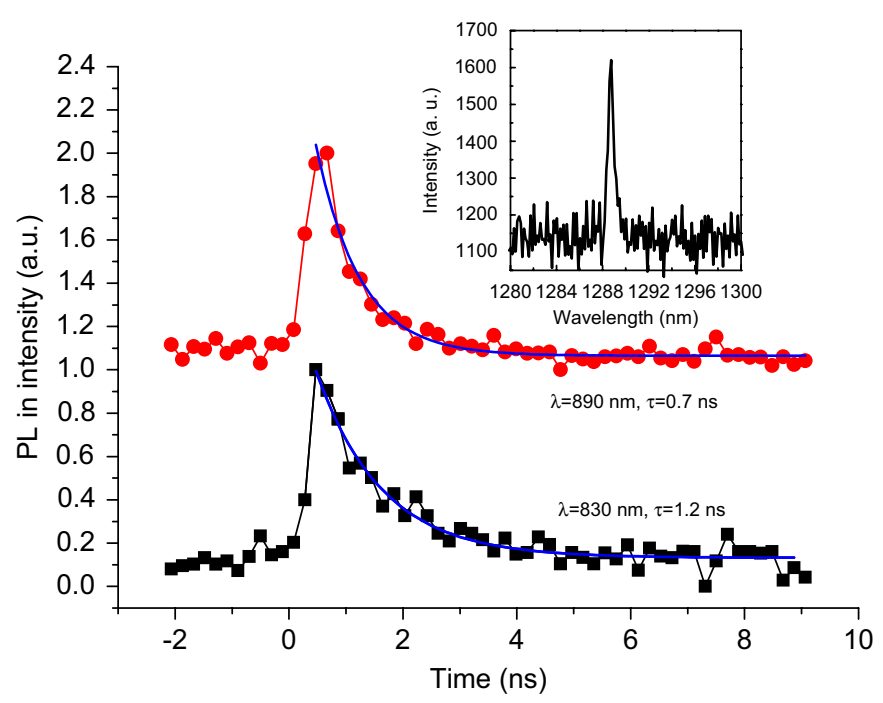

Fig. 3. Single pulse decay with pumping wavelength at 830 and $890 \mathrm{~nm}$. The lines are shifted for clarity. Inset: a typical PL spectrum of an exciton in a quantum dot with an OTF.

resonant pumping has been observed before [13]. In principle, the single photon sources can be operated faster with resonant pumping than non-resonant pumping.

Next we investigate the decay dynamics with two optical pulses. The delay time between the two pulses controlled by transition stage varies from 0.25 to $1.5 \mathrm{~ns}$, corresponding to drive rates between $4 \mathrm{GHz}$ and $667 \mathrm{MHz}$. Fig. 4(a) shows the decay dynamics of a single exciton pumping at $830 \mathrm{~nm}$ with different separations between the two pulses. The two pulse events can be resolved from $1 \mathrm{~ns}$ separation; However, most of them are overlapped. An output ratio has been introduced to show the output photon pulse rate [14], which is the counts produced by the first pulse, measured before the next excitation pulse arrives divided by the total emitted intensity within a single pulse. Fig. 4(b) shows the results with resonant pumping at $890 \mathrm{~nm}$. The single photon intensity from first pulse can be well isolated from the second pulse from a separation of $1 \mathrm{~ns}$. This indicates that the single-photon sources can be operated around $1 \mathrm{GHz}$. Unfortunately, we cannot fit the experiment data for the output ratio with two pulse decay because of the large noise. The source of the noise is due to the pumping light after the OTF. At resonant pumping, it requires more power to achieve same PL intensity and the energy of pumping is close to the energy of the emitted photons. Therefore, it is difficult to filter out the pumping light by the OTF.

In conclusion, we investigated the decay dynamics of single quantum dots at telecommunication wavelengths pumping with two 
a

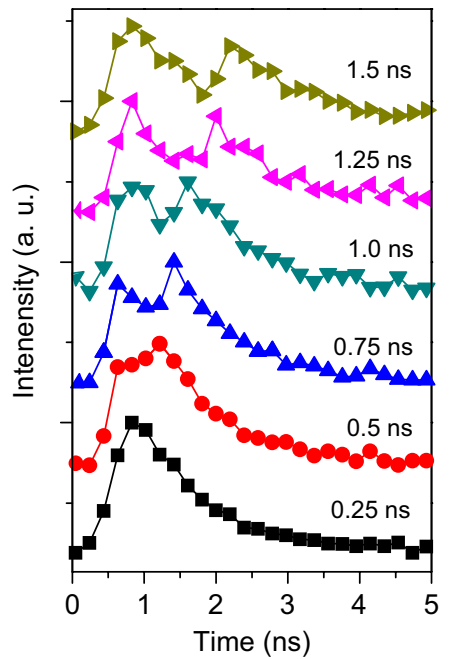

b

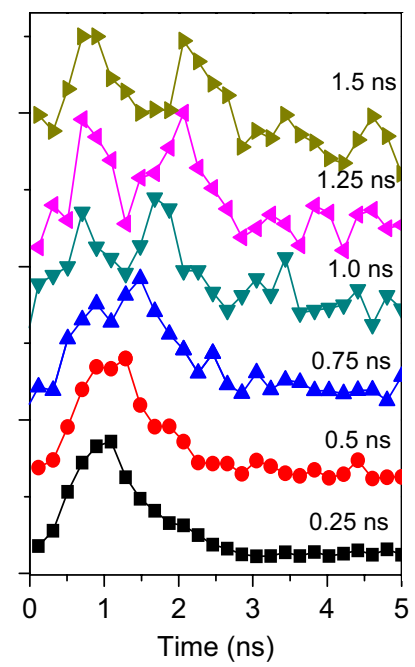

Fig. 4. Time resolved PL measurements with two optical pulses separated from 0.25 to $1.5 \mathrm{~ns}$ with pumping wavelength centered at (a) $830 \mathrm{~nm}$ and (b) $890 \mathrm{~nm}$. The traces are shifted for clarity.

optical pulses resonantly and non-resonantly. With resonant pumping, the lifetime of a single exciton is about $700 \mathrm{ps}$, which is shorter than non-resonant pumping. In principle, this fiber based single photon sources can be operated around $1 \mathrm{GHz}$ at resonant pumping.

This work was partially supported by the Swiss National Science Foundation and the European Commission through the IP "QAP" (Contract no. 15848).

\section{References}

[1] X.L. Xu, D.A. Williams, J.R.A. Cleaver, Appl. Phys. Lett. 85 (2004) 3238.

[2] X.L. Xu, I. Toft, R.T. Phillips, J. Mar, K. Hammura, D.A. Williams, Appl. Phys. Lett 90 (2007) 061103.

[3] Z. Yuan, B.E. Kardynal, R.M. Stevenson, A.J. Shields, C.J. Lobo, K. Cooper, N.S Beattie, D.A. Ritchie, M. Pepper, Science 295 (2002) 102.

[4] P. Michler, A. Kiraz, C. Becher, W.V. Schoenfeld, P.M. Petroff, L. Zhang, E. Hu, A. Imamoglu, Science 290 (2000) 2282.

[5] A. Fiore, C. Zinoni, B. Alloing, C. Monat, L. Balet, L.H. Li, N.L. Thomas, R. Houdre, L. Lunghi, M. Francardi, A. Gerardino, G. Patriarche, J. Phys.: Condens. Matter 19 (2007) 225005

[6] C. Zinoni, B. Alloing, L.H. Li, F. Marsili, A. Fiore, L. Lunghi, A. Gerardino, Y.B. Vakhtomin, K.V. Smirnov, G.N. Gol'tsman, Appl. Phys. Lett. 91 (2007) 031106.

[7] T. Miyazawa, K. Takemoto, Y. Sakuma, S. Hirose, T. Usuki, N. Yokoyama, M. Takatsu, Y. Arakawa, Jpn. J. Appl. Phys. 44 (Part 2) (2005) L620.

[8] S. Kako, C. Santori, K. Hoshino, S. Götzinger, Y. Yamamoto, Y. Arakawa, Nat. Mater. 5 (2006) 887.

[9] X.L. Xu, F. Brossard, K. Hammura, D.A. Williams, B. Alloing, L.H. Li, A. Fiore, Appl. Phys. Lett. 93 (2008) 021124.

[10] V.S.C. Manga Rao, S. Hughes, Phys. Rev. Lett. 99 (2007) 193901.

[11] B. Alloing, C. Zinoni, V. Zwiller, L.H. Li, C. Monat, M. Gobet, G. Buchs, A. Fiore, E. Pelucchi, E. Kapon, Appl. Phys. Lett. 86 (2005) 101908

[12] B. Alloing, C. Zinoni, L.H. Li, A. Fiore, G. Patriarche, J. Appl. Phys. 101 (2007) 024918.

[13] F. Pulizzi, A.J. Kent, A. Patane, L. Eaves, M. Henini, Semicond. Sci. Technol. 19 (2004) S282.

[14] T. Miyazawa, T. Nakaoka, T. Usuki, Y. Arakawa, K. Takemoto, S. Hirose, S. Okumura, M. Takatsu, N. Yokoyama, Appl. Phys. Lett. 92 (2008) 161104. 